\title{
The Nonlinear Viscoelasticity of the Human Knee Articular Cartilage Chao-Jie WAN ${ }^{1}$, Gai-Min LI', and Tong-Tong GUO',* \\ ${ }^{1}$ Nature Science Department, Harbin Institute of Technology Shenzhen Graduate School, 518000Shenzhen, China \\ *tongtong.guo@hitsz.edu.cn
}

Keywords: Human knee joint, Cartilage, Constitutive, Viscoelasticity, Stress relaxation.

\begin{abstract}
The human knee cartilage play an important role in the knee joint. Osteoarthritis (OA) are related to knee joint cartilage. So the accurate description of the cartilage constitutive is the most important work for the understanding the cartilage test phenomenon. The role of viscoelasticity of collagen fibers in bovine articular cartilage was examined in compression and tension using stress relaxation measurements in the axial direction (normal to the articular surface. The human knee joint cartilage also shows the same viscoelastic property in compression and tension test using stress relaxation measurement in the direction (normal to the articular surface). The tension and compression test show the non-linear viscoelastic property. A viscoelastic fibril-reinforced model including fluid flow was used for analysis of the experimental data. The collagen fibrillar matrix was assumed to be viscoelastic with a strain-dependent tensile modulus, and the nonfibrillar matrix was modeled as linearly elastic. For axial tension, collagen viscoelasticity was found to account for most of the stress relaxation, while the effects of fluid pressurization on the tensile stress were negligible. So we use the viscoelastic constitutive to define the cartilage for simulating the tension and compression test. This study illustrates the essential and mainly role of collagen viscoelasticity.
\end{abstract}

\section{Introduction}

Articular cartilage provides a low friction, wear resistant bearing surface in diarthrodial joints and distributes stresses to the underlying bone. The articular cartilage consists of three major structural constituents: collagen fibers, proteoglycan matrix, and interstitial water. Recent developments in mathematical modeling have improved our understanding of cartilage mechanics, such as the high ratio of transient over equilibrium loads, the load-sharing between the solid and fluid phases, and the dependence of the apparent stiffness of the cartilage on loading velocity [1]. Experimentally, for the axial tension and compression test, the cartilage presents the non-linear viscoelastic property. Based on the test, there may be two load bearing mechanisms accounting for the transient mechanical behavior, i.e.a fluid-dependent and fluid-independent [2]. However, the contribution of structural constituents to each mechanism have not been fully investigated theoretically.

Articular cartilage is a stiff, viscoelastic, fibril-composite material. The importance of collagen fibers for the mechanical function and integrity of cartilage has been demonstrated experimentally. Stress relaxation has been observed in tension and compression testing of cartilage, where the viscoelasticity is attributed primarily to the collagen fibers [2]. Many theories to explain articular cartilage behavior under loading, expressed in computational models that consider the nonlinearly tension and compression of the cartilage. There are several model account for the collagen network. (Li et al., 2000, 2002; Li and Herzog, 2004; Wilson et al. 2004b). And these models all include the viscoelastic behavior [3].

In current attempt to study the mechanisms of cartilage in cartilage, the collagen fibrillar matrix was assumed to be viscoelastic, and the nonfibrillar matrix was considered to linear elastic. For the tensile test, the transient response was dominated by collagen viscoelasticity. The most common tests used to determine the mechanical quality of articular are those of tension, confined compression, unconfined compression, indentation [3]. But none of theory models can explain all of the tests phenomenon.

We hypothesized that a constitutive model considering collagen fiber and non-fibrillar matrix, not considering the fluid-dependent mechanism effect. The collagen fiber and non-fibrillar matrix were 
assumed as a whole part, then we assume the whole part as a viscoelastic constitutive model, namely the cartilage is considered as a viscoelastic constitutive model.

\section{Material and Method}

\section{Sample Extraction}

The test data and method of the research are derived from literature. The fresh knee joint cartilage was obtained from the bodies of healthy adult humans ( $n=10$, aged 19-23 years old, typical postmortem time $2 \mathrm{~h}$ ). Full-thickness $(3-6 \mathrm{~mm})$ cartilage layer was removed from the underlying subchondral bone with a razor blade. The samples were made with length $12 \mathrm{~mm}$, width $3 \mathrm{~mm}$, thickness $1 \mathrm{~mm}$. Articular cartilage is same as other soft tissues, the elastic of it mainly from the entropy change. Therefore there is no unique natural state. So we first pre-stressed sample, ie at the same stress level loading and unloading 20 times. Before mechanical testing, the samples were equilibrated in the phosphate-buffered saline (PBS), $\mathrm{pH} 7.4$ [4-6].

\section{Mechanical Testing}

The hexahedral samples $(\mathrm{n}=10)$ and discs samples were tested in tensile and unconfined compression. The mechanical tests were conducted using Shimadzu AUTOGRAPH electronic universal testing machine equipped with automatic control of stress and strain rate to increase and maintain them constant feature. The load is passed through the load cell, the maximum range of the load cell is $200 \mathrm{~N}$ and the using range is $100 \mathrm{~N}$. For the tensile test, the pre-adjusted sample was fixed on the soft tissuespecific specimen clamping fixture, the fixture with the plexiglass cylinder connection, a built aquarium $\mathrm{pH} 7.4$ saline, the sample was placed in the saline. Then the fixture was equipped with a specimen and testing machine, the next chuck connection [4-5]. In the tensile mechanical test, a fully automated series of stress relaxation tests (velocity $1 \mu \mathrm{m} / \mathrm{s}$ ) was up to 20 percent strain. The complete time-position-load data were recorded [7].

In order to perform the unconfined compressive test in the axial direction, samples were glued between loading platens of the testing instrument by using syanoacrylate. The articular surface was then dried with a paper towel, and glued onto the other loading platen by driving the platen into contact with the sample. In the unconfined compressive test, the fully automated series of stress relaxation tests (velocity $1 \mu \mathrm{m} / \mathrm{s}$ ) was reached up to 20 percent strain.

\section{Model Simulation}

\section{Formatting the Title}

Stress relaxation of the knee joint cartilage was simulated numerically by using the same geometry and loading procedures as those used in the tensile and compressive tests (except that the bone was not modeled). The nonlinear viscoelastic model was used, which consisted of collagen fiber and nonfibrillar matrix, not considered the influence of cartilage fluid. The viscoelastic behavior of the cartilage was represented by a linear spring (with spring constant E0), parallel to a non-linear spring (with spring constant) in series with a linear dashpot (with damping coefficient $\eta$ ).

We assume the cartilage property as the homogeneous isotropic nonlinear viscoelastic model (Fig.3). The behavior of one-dimensional model is given as below [8]:

$$
\sigma=-\frac{\eta}{2 \sqrt{\left(\sigma-E_{0} \varepsilon\right) E_{\varepsilon}}} \dot{\sigma}+E_{0} \varepsilon+\left(\eta+\frac{\eta E_{0}}{2 \sqrt{\left(\sigma-E_{0} \varepsilon\right) E_{\varepsilon}}}\right) \dot{\varepsilon}
$$

Where and are the stress and strain of the cartilage respectively. This differential equation was numerically integrated using an implicit backward Euler scheme.

The relaxation function of the model is given as:

$$
\sigma=-\frac{\eta}{2 \sqrt{\left(\sigma-E_{0} \varepsilon_{0}\right) E_{\varepsilon}}} \dot{\sigma}+E_{0} \varepsilon_{0}
$$

The initial condition of the relaxation test is when $t \geq 0, \dot{\varepsilon}=0, \varepsilon=\varepsilon_{0}$.

The creep function of the model is given as: 


$$
\left(\eta+\frac{\eta E_{0}}{2 \sqrt{\left(\sigma-E_{0} \varepsilon_{0}\right) E_{\varepsilon}}}\right) \dot{\varepsilon}+E_{0} \varepsilon_{0}=\sigma_{0}
$$

The initial condition of the creep test is when $t \geq 0, \sigma=\sigma_{0}, \dot{\sigma}=0$.
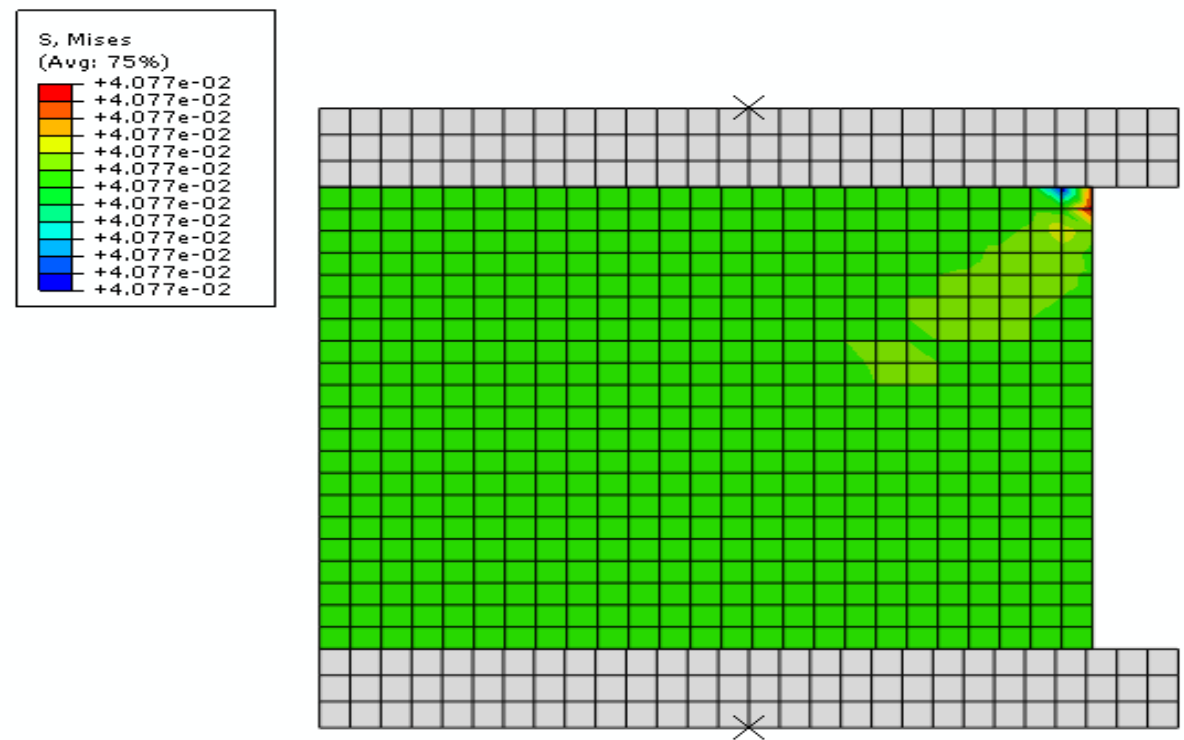

Figure 1. The compression test finite element model.
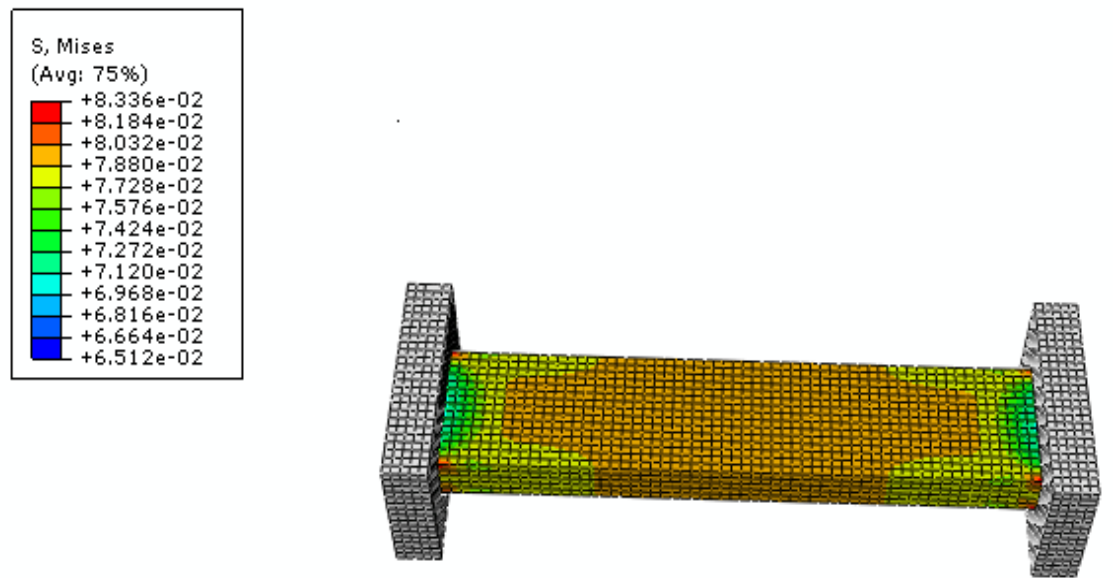

Figure 2.The tension test finite element model

The unknown parameters are $E_{\varepsilon}, E_{0}, \eta, v$ and $v$ was assumed to $0.158 . E_{\varepsilon}, E_{0}$, and $\eta$ were determined by fitting them to the results of tensile and compressive experiment.

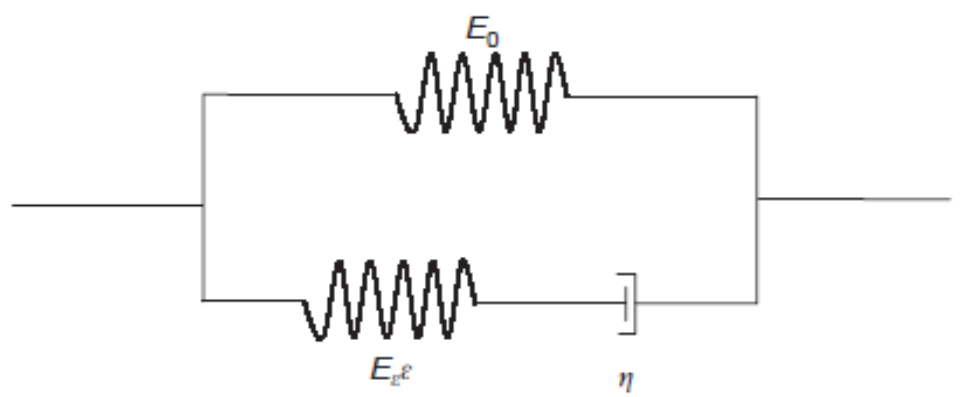

Figure 3. Schematic model for the viscoelastic 
The fitting procedures were performed iteratively, using a multidimensional unconstrained nonlinear minimization procedure available in Matlab Version 7.0. The nonlinear viscoelastic constitutive model was implemented in ABAQUS v6.8. (Hibbitt, Karlsson \& Sorensen, Inc., Pawtucket, RI, USA). The subroutine UMAT was used to define the material behavior of the total solid matrix. An updated Lagrange procedure was used to account for geometric nonlinearities that occurred within this model [7].

For the simulation of the tensile test hexahedral finite element mesh was used (Fig.2). The nodal displacements at the bottom pane were confined in the z-direction. The mesh was axially compressed by $20 \%$, the ramp strain rate of $1 \mu \mathrm{m} / \mathrm{s}$ was applied. The strain was held constant until full relaxation occurred and the axial forces were computed.

In order to the simulation of the compressive test an axisymmetric finite element was used (Fig.1). The nodal displacements at the bottom plane were confined in the z-direction. The displacements of the nodes at the symmetry axis were confined in the r-direction. The ramp strain rate of $1 \mu \mathrm{m} / \mathrm{s}$ was applied, when the axial strain reached $20 \%$, holding the strain constant until the full relaxation occurred [6].

\section{Results}

The typical stress relaxation response from the experiment and the theoretical model prediction, for the cartilage disk and hexahedral subjected to the axial compression and tension, respectively. During the relaxation time, the axial reaction force of the tension and compression is determined by the constitutive model and the cross-sectional area of the samples. The results from the model predictions was using the average cartilage tissue properties defined the nonlinear viscoelastic property. The results of simulating relaxation experiment are almost in perfect agreement with the experimental data (Fig.4 and Fig.5). The experimental data obtained from the disk sample is derived from the literature of J S Jurvelin and M D Buschmann and E B Hunziker (2003). The tensile experimental data is derived from the literature of ZHAO Baolin and ZHANG Zhongjun and MA Hongshun (2005). The result of the simulation show that assuming the constitutive of knee joint cartilage as homogeneous, isotropic and nonlinear viscoelastic model is reasonable.

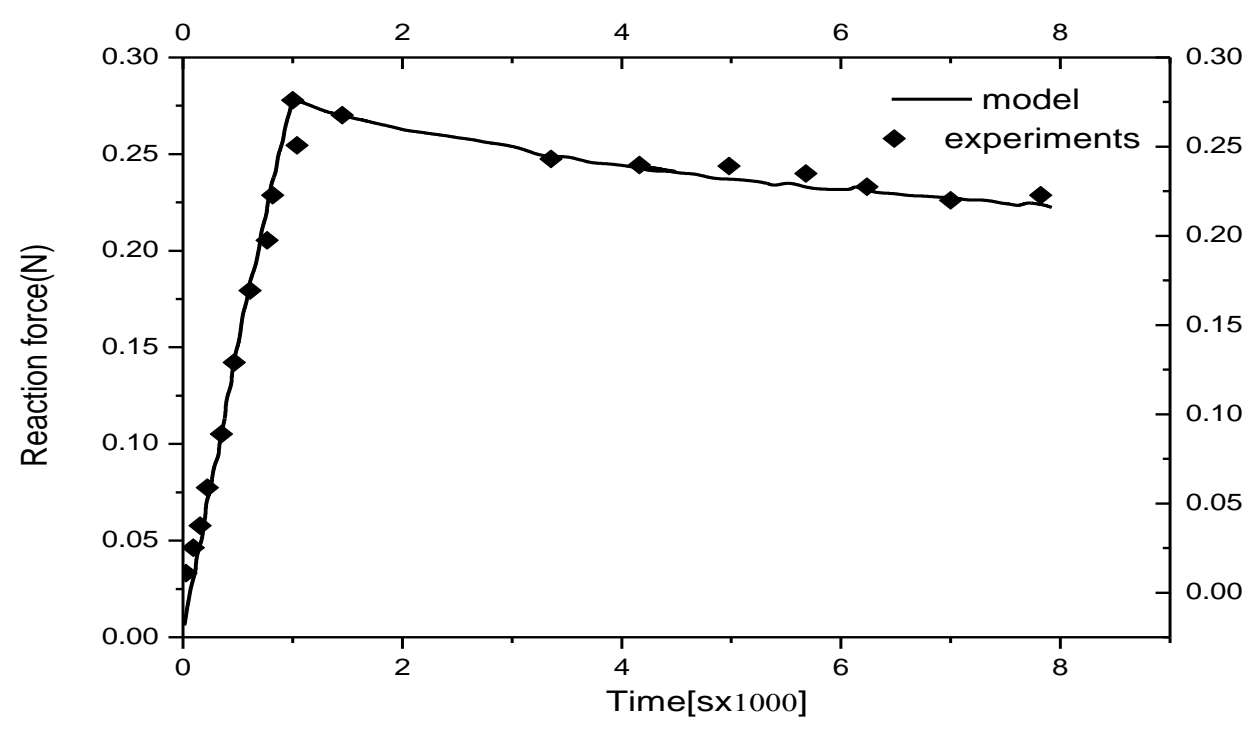

Figure 4. Axial reaction force, measured from the tension stress relaxation test. 


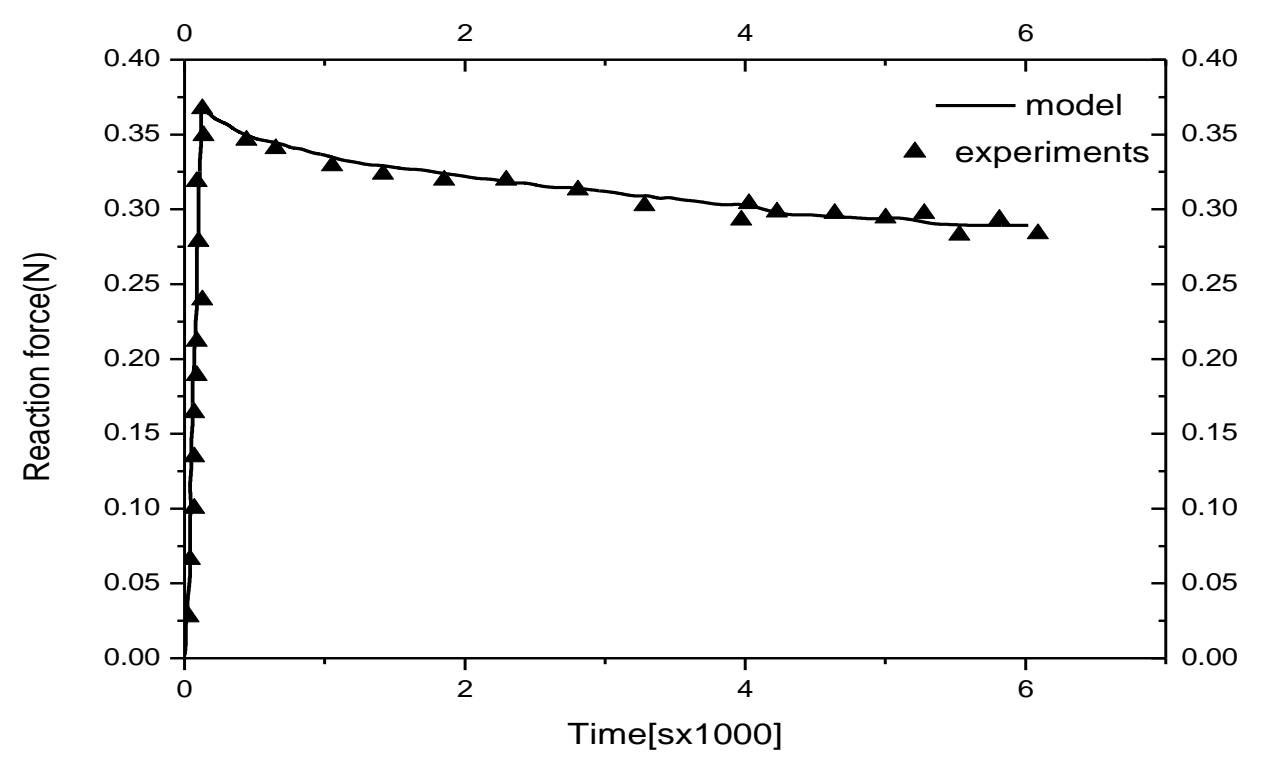

Figure 5. Axial reaction force, measure from the compression stress relaxation test.

\section{Discussion}

In the present study, the stress relaxation response of human knee joint cartilage in the axial compression and tension was experimentally measured and numerically modeled. Observed from the experimental data and phenomenon, the cartilage tissue exhibited considerable stress relaxation for the axial tensile and compressive. In the theoretical model, the constitutive model of the cartilage was represented well with the collagen fiber and non-fibrillar matrix considered as viscoelasticity, not considering the influence of the fluid flow. However, some of researches demonstrated that the permeability of the cartilage has the effect on the confined compression, unconfined compression and indentation test. Recently, the most widely accepted theoretical model was applied, i.e. the linear biphasic model of cartilage [11] for the analysis of experimental confined compression measurements. To justify the use of the model the authors attempted to satisfy the model assumptions as well as possible. The most superficial and deepest tissue was excluded to obtain more homogeneous samples with an isotropically oriented collagen network. Also, the study was limited to the use of a low ramp speed $(1 \mu \mathrm{m} / \mathrm{s})$. However, the theory did not perfectly agree with the experimental measurements (Fig.4 and Fig.5).

In summary, the study characterizes homogeneous isotropic nonlinear viscoelastic mechanical of adult human knee joint cartilage in tension and compression.

\section{Acknowledgment}

This study was acknowledged by NSFC (10872061).

\section{References}

1. Lorna Edelsten, Janet E. Jeffrey, Leanne V. Viscoelastic properties of articular cartilage subjected to impact loading in vitro. Journal of Biomechanics 41(S1).

2. L.P. Li, W. Herzog, R.K. Korhonen, J.S. Jurvelin. The role of viscoelasticity of collagen fibers in the articular cartilage: axial tension versus compression. Medical Engineering \& Physics 27 , $51-$ 57(2005). 
3. W. Wilson, C.C. van Donkelaar, B. van Rietbergen, R. Huiskes. A fibril-reinforced poroviscoelastic swelling model for articular cartilage. Journal of Biomechanics 38 ,11951204(2005).

4. SUN Changjiang, FENG Tiejian, LU Yaping, MA Hongshun. The Experimental Study of Tracheal Cartilage Stress Relaxation. Journal of Biomedical Engineering Research 2010,29 (1) :3739.

5. ZHAO Baolin, ZHANG Zhongjun, MA Hongshun. Biomechanical Properties of the Cartilage of Shoulder Joint, Hip Joint and Knee Join. Beijing Biomedical Engineering 2005, Vo1.24 No.3(2005).

6. J S Jurvelin, M D Buschmann, E B Hunziker. Mechanical anisotropy of the human knee articular cartilage in compression. Proc. InstnMech. Engrs Vol. 217 Part H: J. Engineering inMedicine(2003).

7. W. Wilson, C.C. van Donkelaar, B. van Rietbergen, K. Ito, R. Huiskesa. Stresses in the local collagen network of articular cartilage: a poroviscoelastic fibril-reinforced finite element study. Journal of Biomechanics 37, 357-366(2004).

8. W. Wilson, C.C. van Donkelaar, B. van Rietbergen, R. Huiskes. Erratum to "Stresses in the local collagen network of articular cartilage: a poroviscoelastic fibril-reinforced finite element study" [Journal of Biomechanics 37 (2004) 357-366] and "A fibril-reinforced poroviscoelastic swelling model for articular cartilage" [Journal of Biomechanics 38 (2005) 1195-1204]. Journal of Biomechanics 38 (2005) 2138-2140. 